\title{
Digital modulation identification in terms of software defined radio for traffic management system
}

\begin{abstract}
Nowadays, a lot of efforts have been given to reduce hardware and software development cost. Many of today's military and commercial communication systems use Software Defined Radio (SDR) technology to create various different hardware combinations in term of software control. In this paper, we present an approach to design a SDR based traffic management system by tuning between two different digital modulation schemes: ASK and FSK. The wavelet transform technique has been used for modulation identification. The performance of the identification scheme is investigated through simulations, for different signal to noise ratio (SNR). The system identification performance is improved more than $95 \%$ when SNR is more than $9 \mathrm{~dB}$.
\end{abstract}

Keyword: Component; Digital modulation; Software defined radio; ASK; FSK 